\title{
Emergence of human rotavirus genotype G9 in metropolitan Detroit between 2007 and 2009
}

\section{Correspondence \\ Nahed Abdel-Haq nabdel@dmc.org}

Received 11 October 2010 Accepted 1 March 2011

\author{
Nahed Abdel-Haq, ${ }^{1,2}$ Muhammad Amjad, ${ }^{3}$ Eric McGrath, ${ }^{1,2}$ \\ Pimpanada Chearskul, ${ }^{2}$ Ahdi Amer, ${ }^{2}$ Hossein Salimnia ${ }^{4,5}$ \\ and Basim I. Asmar ${ }^{1,2}$ \\ ${ }^{1}$ Division of Infectious Diseases, Children's Hospital of Michigan, Detroit, MI, USA \\ ${ }^{2}$ Carman and Ann Adams Department of Pediatrics, Wayne State University, Detroit, MI, USA \\ ${ }^{3}$ Department of Clinical Laboratory Sciences, Marshall University, Huntington, WV, USA \\ ${ }^{4}$ Department of Pathology, Wayne State University, Detroit, MI, USA \\ ${ }^{5}$ Detroit Medical Center University Laboratories, Detroit, MI, USA
}

\begin{abstract}
Between January 2007 and April 2009, rotavirus (RV)-positive stool samples from 238 children with acute gastroenteritis, seen at Children's Hospital of Michigan in Detroit, USA, were collected and RV genotyping was performed. $G$ and $P$ genotypes were determined by RT-PCR and nucleotide sequencing was conducted on selected $G 9$ and $P[6]$ strains. Correlation between the severity of gastroenteritis episode and the infecting $G$ genotype was done using a 14-point scoring system. The predominant G genotype was G9 (39.5\%), followed by G1 (35.3\%) and G4 (15.5\%), while $P[8]$ was the most prevalent $P$ genotype (66.5\%), followed by $P[4](21.9 \%)$ and $\mathrm{P}[6]$ (11.2\%). The gene combinations G1P[8] and G9P[8] were the most prevalent (21.4\% and $20.6 \%$, respectively), followed by G4P[8] (13\%) and G9P[6] (8.8\%). Immunization data showed that only $17 / 238(7.1 \%)$ children received $\geqslant$ one dose of RV vaccine (the pentavalent vaccine RotaTeq or the monovalent vaccine Rotarix) and that 10/17 were infected with G4P[8] strains. Severity of RV gastroenteritis episodes was not related to the infecting $G$ genotype. Our results suggest a high proportion of genotype $G 9$ strains in combination with $P[8], P[6]$ and $P[4]$ specificity circulating in the metropolitan Detroit area. While the protective efficacy of the RV vaccines has been demonstrated against G9P[8] strains, the level of cross-protection offered by the vaccines against $G 9$ strains with $P[6]$ and $P[4]$ genotypes in the Detroit paediatric population remains to be determined.
\end{abstract}

\section{INTRODUCTION}

Rotavirus (RV) is a major cause of acute gastroenteritis worldwide. It accounts for approximately $40 \%$ of diarrhoea hospitalizations in children $<5$ years of age (Parashar et al., 2006). An estimated 527000 RV-infected children younger than 5 years die each year, the majority in developing countries (Parashar et al., 2003). Control of RV disease is most likely to be achieved through widespread use of an effective vaccine.

The outer layer of group A RV is composed of two proteins, VP4 and the VP7, which are encoded by RNA segment 9 and 4, respectively (Estes \& Kapikian, 2007). Rotaviruses are classified into $\mathrm{G}$ and $\mathrm{P}$ types based on the reactivity of the

Abbreviation: CDC, Centers for Disease Control and Prevention.

The GenBank/EMBL/DDBJ accession numbers for the VP7 gene sequence of G9 strains isolated in this study are HQ018932, HO018933 and HO018934. outer capsid proteins, VP7 and VP4, with serotype-specific monoclonal antibodies or on the amplification of PCR amplicons using genotype-specific primers. Genotype $\mathrm{P}[8]$ is the most common P type globally, followed by $\mathrm{P}[4]$ and $\mathrm{P}[6]$. $\mathrm{P}[8]$ tends to be more frequently associated with serotypes G1, G3 and G4, while P[4] is more likely to be associated with serotype G2. Prior to 1990, the most common serotypes globally were G1P[8], G2P[4], G3P[8] and G4P[8]; since then, G9P[8] has been recognized as the fifth most globally common type (Gentsch et al., 1992, 2009; Koshimura et al., 2000). The $G$ and $P$ type specific antibodies, particularly those to the $\mathrm{G}$ antigen, are believed to play an important role in protection against RV gastroenteritis (Gentsch et al., 2005). This prompted the development of vaccines based on the most common circulating strains in target populations at the time: G1, G2, G3 and G4. Although the first licensed RV vaccines were designed to cover the most common $G$ types, the current RV vaccines were designed to cover the most common G and P types (Gentsch et al., 2005). 
The RV vaccine strategy is based on eliciting immunity in recipients comparable to that induced by natural infection through production of homotypic and heterotypic antibodies to serotypes targeted by the vaccine. Presently, two oral RV vaccines are approved by the Federal Drug Administration of the USA for administration to infants. A pentavalent human-bovine reassortant vaccine, Rotateq, was developed by Merck and contains the four major $G$ serotypes G1, G2, G3 and G4 with the most predominant P type, $\mathrm{P}[8]$ (RV5). A monovalent attenuated human vaccine, Rotarix, was developed by GlaxoSmithKline Biologicals and contains a G1P[8] strain (RV1).

Studies from different parts of the world suggest that serotypes other than G1-G4 are important causes of RV gastroenteritis. These include G5 in Brazil and G8 in Malawi and other parts of Africa (Cunliffe et al., 2001; Leite et al., 1996). G9 strains emerged in 1993 as one of five globally prevalent genotypes and in 2001 accounted for a rate of at least $5.8 \%$ of RV cases (Laird et al., 2003). However, this may not reflect the current prevalence of the G9 genotype, since recent regional epidemiological studies have shown prevalence of G9 genotypes as high as 50-90\% in some settings (de Rougemont et al., 2009; Rodrigues et al., 2007; Sánchez-Fauquier et al., 2006).

In 2001, we genotyped RV strains recovered from 100 children with acute gastroenteritis seen at the Children's Hospital of Michigan in Detroit, MI, USA. G1 was the predominant genotype (73\%), followed by G4 (17.8\%). Genotype G9, not present in the current vaccine formulations, was detected at very low frequencies in the USA in the late 1990s and in the Detroit study in $7 \%$ of patients (Abdel-Haq et al., 2003). Whether widespread implementation of RV vaccines will cause emergence of nonvaccine serotypes was unclear.

Genotyping of circulating RV strains has important implications on the protective efficacy of the current vaccine formulations and monitoring of vaccine impact. Although natural RV infection in infants confers protection against subsequent infection (Velázquez et al., 1996), $R V$ vaccines are most effective when they include viral serotypes similar to those circulating in the community (homotypic responses). Vaccines with non-matching circulating serotypes induce heterotypic responses that are believed to be less protective (Perez-Schael et al., 1990). This study was undertaken at the Children's Hospital of Michigan to monitor the prevalence of RV genotypes in metropolitan Detroit and to detect possible replacement of the predominant serotypes following RV vaccine licensing in February 2006. We also studied the correlation between the specific causative RV genotype and severity of gastroenteritis episodes.

\section{METHODS}

Patients. Between January 2007 and April 2009, stool specimens were collected from infants and children under 6 years of age presenting with acute gastroenteritis to the Children's Hospital of Michigan. The hospital has a 230-bed capacity and is a tertiary care teaching institution. Stools were tested for RV antigen using the enzyme immunoassay detection kit Sure-Vue Rota Test (Fisher HealthCare), and stored at $-80{ }^{\circ} \mathrm{C}$ in $10 \%$ balanced salt solution until RNA extraction. The study was approved by the Human Investigation Committee at Wayne State University.

RNA extraction, and G and P typing by RT-PCR. RV RNA from stool samples was extracted using TRIzol Reagent according to the manufacturer's recommendation (Sigma-Aldrich). RNA was stored at $-80{ }^{\circ} \mathrm{C}$ in nuclease-free water until further use. Reverse transcription was performed by using Ready-to-Go RT-PCR beads (GE Healthcare) using random hexamer primers.

RV G and P types were determined using two-step, primer-specific, semi-nested multiplex PCR. For G typing, PCR amplification was performed using VP7 forward and VP7 reverse primers. The second step multiplex PCR was performed using VP7 reverse and G1, G2, G3, G4, G9 and G10 specific primers (Gouvea et al., 1990; Iturriza-Gómara et al., 2004). P typing was also performed as a two-step PCR employing Con 2 and Con 3 for the first PCR for amplification of the VP4 segment, and Con3, P[4], P[6], P[8], P[9], P[10] and P[11] specific primers for the second multiplex PCR (Gentsch et al., 1992; Iturriza-Gómara et al., 2004). PCR amplifications were performed by using PCR reagents (Bio-Rad) and amplification conditions described previously (Iturriza-Gómara et al., 2004). The resulting amplicons were analysed on a $2 \%$ agarose gel stained with ethidium bromide. $\mathrm{G}$ and $\mathrm{P}$ genotypes were determined on the basis of amplicon sizes (Gentsch et al., 1992; Gouvea et al., 1990; Iturriza-Gómara et al., 2004).

Sequence alignments and phylogenetic analysis. Representative G9 and P[6] strains were sequenced by using the primers Beg9 and End9 to sequence G types (Gouvea et al., 1990), and the primers Con2 and Con3 to sequence P types (Gentsch et al., 1992). The PCR products were purified using a Spin Column Gel Extraction kit (Qiagen) and used as template for sequencing by Big-Dye Terminator cycle sequence reaction in an automated ABI DNA sequence analyser (Applied Biosystems). Multiple alignments and phylogenetic analysis were performed using UPGMA and neighbour-joining methods using CLC sequence analysis software (CLC bio).

Clinical data. Clinical data were collected retrospectively. A 14-point scoring system modified by Cascio and colleagues was used to classify the severity of RV disease as shown in Table 1 (Ansaldi et al., 2007; Cascio et al., 2001). RV vaccination status was recorded by accessing the Michigan Care Improvement Registry.

Statistical analysis. Statistical analyses of clinical variables and genotype frequencies were performed using sPSs version 17.0. The non-parametric Kruskal-Wallis one-way analysis of variance by ranks procedure was employed for testing equality of population medians of severity scores among genotype groups, followed by pairwise examinations using the non-parametric Mann-Whitney test for unpaired data. In all comparisons, statistically significant differences were considered achieved at a $P$-value $\leqslant 0.05$, two-tailed.

\section{RESULTS}

For the 36-month study period between 2007 and 2009, RV was detected by enzyme immunoassay in 304 stool samples in 2007, in 95 stool samples in 2008 and in 230 stool samples in 2009. A total of 238 (38\%) RV-positive stool samples were randomly selected from 2007-2009 and genotyped. The age range of these children was 11 days-70 
Table 1. Classification system used to score RV gastroenteritis disease severity

\begin{tabular}{|llc|}
\hline Symptom & \multicolumn{1}{c}{ Occurrence } & Score \\
\hline Diarrhoea - duration & $<2$ days & 1 \\
& $2-4$ days & 2 \\
Diarrhoea - number of stools & $>4$ days & 3 \\
& Three stools & 1 \\
& Four to five stools & 2 \\
Vomiting and duration & $>$ Five stools & 3 \\
& None & 0 \\
Fever (temperature $\left.>38.2{ }^{\circ} \mathrm{C}\right)$ & $1-2$ days & 1 \\
& $>2$ days & 3 \\
Intravenous hydration required & No & 0 \\
& No & 2 \\
& Yes & 0 \\
& & 3 \\
\hline
\end{tabular}

months (mean 18 months, median 16 months). Of these, $57 \%$ were boys and $36 \%$ were younger than 1 year.

Over the study period, G9 was the predominant G genotype $(39.5 \%)$, followed by G1 (35.3\%), G4 (15.5\%), G3 (5.4\%) and G2 (4.3\%). Of the VP4 genotypes detected, $\mathrm{P}[8]$ was the most prevalent $(66.5 \%)$, followed by $\mathrm{P}[4]$ $(21.9 \%)$ and $\mathrm{P}[6](11.2 \%)$. The distribution of $\mathrm{G}$ and $\mathrm{P}$ genotypes for each year is shown in Table 2. Of the 102 strains with G9 genotypes, the majority $(63 \%)$ were associated with P[8] followed by P[6] and P[4]. All 11 G2 genotypes were associated with the $\mathrm{P}[4]$ genotype (Table 3 ).

The most prevalent G-P combination was G1P[8] (21.4\%), followed by G9P[8] (20.6\%) and G4P[8] (13\%). Twenty patients were infected with two G types, $18 \mathrm{G} 1+\mathrm{G} 9$ and two G4 + G9, and four with two P types, three $P[4]+P[6]$ and one $\mathrm{P}[8]+\mathrm{P}[6]$. All mixed infections with more than one $\mathrm{G}$ genotype included G9 strains. One stool sample contained
$\mathrm{P}$ [9] genotype and additional testing is being conducted to verify the result. The distribution and frequency of $G$ and $P$ genotype combinations are shown in Table 3.

Representative PCR products of G9 and P[6] genotypes were verified by nucleotide sequencing. The VP7 sequences of 2007, 2008 and 2009 strains isolated during this study showed $>99 \%$ similarity to each other, and $>97 \%$ similarity to the reference strain AF060487 (US 1205/ Indiana/US). Sequences were submitted to GenBank (accession nos HQ018932, HQ018933 and HQ018934). Sequence alignment and phylogenetic analyses of the VP7 gene of G9 strains isolated during this study demonstrated that these strains belong to the VP7 lineage 3 (Fig. 1).

Data on the RV immunization status were available from the Michigan Care Improvement Registry for 372 patients seen during the study period and revealed that only 33 $(8.9 \%)$ had received $\geqslant$ one dose of RV vaccine (RV5 or RV1). Of those, only $9 / 33$ (27.3\%) received three doses of RV5. Only $17(7.1 \%)$ of the 238 children included in the study had received $\geqslant$ one dose of RV5 or RV1; 10/17 were infected with G4P[8] strains.

Using the 14-point scoring system, we evaluated the severity of clinical symptoms of RV gastroenteritis in 125 patients who had complete clinical data available. These included 36 patients infected with G1 strains, 9 with G2, 11 with G3, 19 with G4, 40 with G9 and 10 with G1 + G9 strains. The mean scores in each group were 10.56, 9.78, 11.1, 11.3, 10.8 and 12.6, respectively. Differences among means of severity scores were not statistically significant $(P>0.05)$; however, groups were unbalanced in sample size.

\section{DISCUSSION}

To our knowledge, this is the first report from the USA identifying the G9 genotype as the predominant circulating

Table 2. Distribution and frequency of $G$ and $P$ genotypes of $R V$ detected in Detroit from 2007 to 2009

\begin{tabular}{|c|c|c|c|c|c|c|c|c|}
\hline \multirow[t]{2}{*}{ Genotype } & \multicolumn{2}{|c|}{2007} & \multicolumn{2}{|c|}{2008} & \multicolumn{2}{|c|}{2009} & \multirow[t]{2}{*}{ Total $n^{\star}$} & \multirow[t]{2}{*}{$\%$ over the 3 -year period } \\
\hline & $n$ & $\%$ & $n$ & $\%$ & $n$ & $\%$ & & \\
\hline G2 & 5 & 5.8 & 0 & 0 & 6 & 4.3 & 11 & 4.3 \\
\hline G3 & 2 & 2.3 & 1 & 2.9 & 11 & 8.0 & 14 & 5.4 \\
\hline G4 & 15 & 17.4 & 4 & 11.8 & 21 & 15.2 & 40 & 15.5 \\
\hline $\mathrm{P}[4]$ & 25 & 32.1 & 9 & 26.5 & 19 & 14.6 & 53 & 21.9 \\
\hline $\mathrm{P}[6]$ & 6 & 7.7 & 6 & 17.6 & 15 & 11.5 & 27 & 11.2 \\
\hline $\mathrm{P}[8]$ & 47 & 60.2 & 19 & 55.9 & 95 & 73.1 & 161 & 66.5 \\
\hline $\mathrm{P}[9]$ & 0 & 0 & 0 & 0 & 1 & 0.8 & 1 & 0.4 \\
\hline Total & 78 & 100.0 & 34 & 100.0 & 130 & 100.0 & 242 & 100.0 \\
\hline
\end{tabular}

${ }^{\star}$ The totals exceed the number of specimens analysed because of mixed infections. 
Table 3. Distribution and frequency of $G$ and $P$ genotype combinations of RV detected in Detroit from 2007 to 2009

\begin{tabular}{|c|c|c|c|c|c|c|c|c|}
\hline \multirow[t]{2}{*}{ Genotype $^{*}$} & \multicolumn{2}{|c|}{2007} & \multicolumn{2}{|c|}{2008} & \multicolumn{2}{|c|}{2009} & \multirow[t]{2}{*}{ Total $n$} & \multirow[t]{2}{*}{$\%$ over the 3 -year period } \\
\hline & $n$ & $\%$ & $n$ & $\%$ & $n$ & $\%$ & & \\
\hline $\mathrm{G} 1 \mathrm{P}[4]$ & 11 & 14.1 & 7 & 20.6 & 2 & 1.6 & 20 & 8.4 \\
\hline G1P $[8]$ & 18 & 23.0 & 7 & 20.6 & 26 & 20.6 & 51 & 21.4 \\
\hline $\mathrm{G} 2 \mathrm{P}[4]$ & 5 & 6.4 & 0 & 0 & 6 & 4.8 & 11 & 4.6 \\
\hline G3P[8] & 2 & 2.6 & 1 & 2.9 & 10 & 7.9 & 13 & 5.5 \\
\hline G3P[9] & 0 & 0 & 0 & 0 & 1 & 0.8 & 1 & 0.4 \\
\hline G4P[4] & 5 & 6.4 & 0 & 0 & 0 & 0 & 5 & 2.1 \\
\hline G4P[6] & 0 & 0 & 0 & 0 & 2 & 1.6 & 2 & 0.8 \\
\hline G4P $[8]$ & 9 & 11.5 & 4 & 11.8 & 18 & 14.3 & 31 & 13.0 \\
\hline G9P[4] & 2 & 2.6 & 2 & 5.9 & 6 & 4.8 & 10 & 4.2 \\
\hline G9P[6] & 6 & 7.7 & 6 & 17.6 & 9 & 7.1 & 21 & 8.8 \\
\hline G9P[8] & 12 & 15.4 & 7 & 20.6 & 30 & 23.8 & 49 & 20.6 \\
\hline $\mathrm{G} 1 \mathrm{P}[4+6]$ & 0 & 0 & 0 & 0 & 1 & 0.8 & 1 & 0.4 \\
\hline $\mathrm{G} 1+9 \mathrm{P}[4]$ & 2 & 2.6 & 0 & 0 & 2 & 1.6 & 4 & 1.7 \\
\hline $\mathrm{G} 1+9 \mathrm{P}[8]$ & 5 & 6.4 & 0 & 0 & 9 & 7.1 & 14 & 5.9 \\
\hline $\mathrm{G} 4+9 \mathrm{P}[4]$ & 1 & 1.3 & 0 & 0 & 0 & 0 & 1 & 0.4 \\
\hline $\mathrm{G} 4+9 \mathrm{P}[8]$ & 0 & 0 & 0 & 0 & 1 & 0.8 & 1 & 0.4 \\
\hline $\mathrm{G} 9 \mathrm{P}[4+6]$ & 0 & 0 & 0 & 0 & 2 & 1.6 & 2 & 0.8 \\
\hline G9P $[6+8]$ & 0 & 0 & 0 & 0 & 1 & 0.8 & 1 & 0.4 \\
\hline Total & 78 & 100.0 & 34 & 100.0 & 126 & 100.0 & 238 & 100.0 \\
\hline
\end{tabular}

*The most prevalent strains of the period are highlighted in bold.

RV genotype after vaccine introduction. Serotype G9 was first detected in the USA in 1983 and later in other parts of the world (Clark et al., 1987). In a Centers for Disease Control and Prevention (CDC) surveillance study of more than 3100 strains from 12 US cities conducted prior to the introduction of RV vaccines (1996-2005), G1 genotype was the most prevalent $G$ genotype in most cities in most years accounting for $50-100 \%$ of tested strains (Gentsch et al., 2009). Detection of G9 strains at the same or higher rate than G1 strains occurred only periodically and in cities submitting low numbers of stool samples, such as in Denver during 1999-2000 when $50 \%$ of 34 samples were G9 and in Atlanta during 2003-2004 when $62 \%$ of 26 submitted stool samples were G9 (Gentsch et al., 2009). In Kansas City during 2002-2003, G9 genotype was detected in 33\% of tested stool samples (Gentsch et al., 2009). Previous studies from the USA and metropolitan Detroit revealed that G9 strains were often detected at low prevalence rates and in association with mixed G genotypes, particularly G1 (AbdelHaq et al., 2003; Ramachandran et al., 1998). In 2001, our detection rate of G9 infections was $6.3 \%$ and all were components of mixed infections (Abdel-Haq et al., 2003). However, during this study (2007-2009), the overall percentage of G9 infections was almost $40 \%$ and more than $77 \%$ of G9 strains were not associated with other genotypes. Sustained high prevalence of G9 strains suggests that G9 may have established itself as a major genotype in our patient population.

Among the different G-P combinations, the previously uncommon strain G9P[8] was an important cause of RV diarrhoea in Detroit during the study period. Before the vaccines became available, global rates of G1P[8], G2P [4] and G9P[8] strains were $64.7 \%, 12 \%$ and $2.7 \%$, respectively (Santos \& Hoshino, 2005). In our present study, the respective rates were $21.4 \%, 4.6 \%$ and $20.6 \%$. In a review of the global distribution of $>21000 \mathrm{RV}$ serotypes from 35 countries, the four common strains G1P[8], G2P[4], G4P[8] and G3P[8] represented $72 \%$ of all isolates (Gentsch et al., 2005 ) in contrast to only $44.5 \%$ in our study. In the CDC survey (1996-2005), the most prevalent strain was G1P[8] (78.5\%) (Gentsch et al., 2009). G9P[8] strains accounted for only $3.6 \%$ in the survey but during the current study was the second most common strain from 2007 to 2009 . Other recent studies indicate that G9P[8] strains are increasing in prevalence globally (CDC, 2008; de Rougemont et al., 2009; Endara et al., 2007; Esteban et al., 2010; Samajdar et al., 2008). In global surveillance studies conducted by the World Health Organization during the period 2001-2008, the most common genotypes were G1, G2, G3, G4 and G9. Excluding the regions of Africa and the Eastern Mediterranean, where the $G$ and $P$ types of a majority of strains could not be determined, the most common strains in all regions were G1P[8], G9P[8] and G2P[4], accounting for approximately two-thirds of the strains with mean detection rates of $28.3 \%, 23.3 \%$ and $16.3 \%$, respectively (CDC, 2008).

In general, G9 strains have been associated with $\mathrm{P}[6], \mathrm{P}[8]$, P[11] or P[19] (Araújo et al., 2001; Santos et al., 2001). G9P[6] was the most common genotype combination found with the first emergence of G9 strains in 1995 


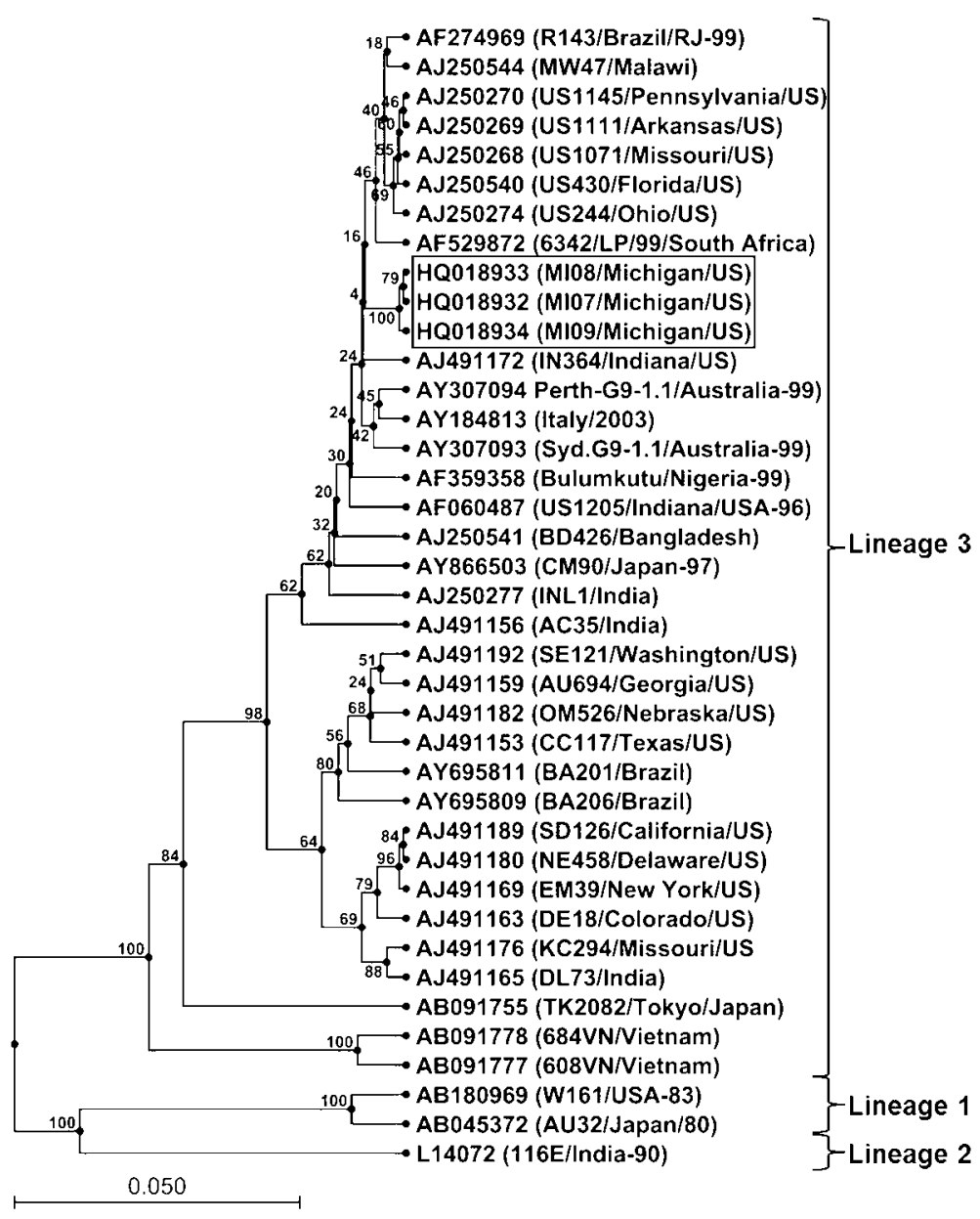

Fig. 1. Phylogenetic analysis of the VP7 gene nucleotide sequence of human RV G9 strains. The dendrogram was constructed by the UPGMA method. Confidence values based on 100 bootstrap replicates are shown on the branches of the tree. Sequences reported in this study along with the GenBank accession numbers are indicated in the highlighted box.
(Iturriza-Gómara et al., 2000). However, more recent studies suggest that G9P[8] is a more common G-P combination (CDC, 2008; de Rougemont et al., 2009; Esteban et al., 2010). In our patients, G9 serotype was associated with $\mathrm{P}[8], \mathrm{P}[6]$ and, less frequently, $\mathrm{P}[4]$. $\mathrm{P}[6]$ is reported as the third most common $\mathrm{P}$ type worldwide and is more prevalent in Africa, Latin America and Asia than in Europe and North America (Gentsch et al., 2005; Steele \& Ivanoff, 2003). In our study, P[6] was detected in 27 patients, representing $11.2 \%$ of all $\mathrm{P}$ genotypes, and was found in combination with 25 G9 genotypes.

The G9 strains sequenced from the study clustered in G9 VP7 lineage 3 (Fig. 1), consistent with the globally emerging G9 strains. The G9 strains were closely related to the strains isolated in USA during the 1993-2001 RV epidemic seasons (Laird et al., 2003; Ramachandran et al., 2000). G9 (lineage 3) strains include some recently isolated strains from Europe, North and South America, Australia and Asia. Lineage 3 also includes some older Vietnamese and Japanese G9 strains (Ansaldi et al., 2007; Martella et al., 2004; Santos et al., 2005). Nucleotide variations in the lineage 3 of recently isolated G9 strains from various parts of the world ranged from $0.6 \%$ to $2.4 \%$, whereas it was 3-5\% for the Vietnamese and Japanese strains (Martella et al., 2004).
G9 and P[6] are not included in the current RV approved vaccines. The efficacy of the current RV vaccines against RV types involving G9 is still to be determined. The monovalent vaccine (Rotarix) is based on the concept of heterotypic protection between serotypes caused by crossreactive antigens while the pentavalent vaccine (RotaTeq) protective efficacy is based on serotype-specific immunity (Gentsch et al., 2005). Despite the fact that G9 is not present in either vaccine formulation, both vaccines have shown protection against G9 strains in previous clinical trials (Ruiz-Palacios et al., 2006; Vesikari et al., 2006). This is probably due to the presence of the $\mathrm{P}[8]$ antigen in both vaccines and the fact that most of the G9 strains circulating in the vaccine trial sites were G9P[8]. However, RotaTeq vaccine protection against G9P[6] strains, which share no antigens with the vaccine strains, could be a major challenge. Similar concerns also apply to the Rotarix vaccine. Although this vaccine may provide protection against strains with different $G$ types associated with $P[8]$, this protection may be challenged by strains that belong to genogroups unrelated to the vaccine strain, such as G9P[6] and G2P[4] (Gentsch et al., 2005; Gurgel et al., 2007).

The RV immunization rate of the patients in our study was very low $(<10 \%)$. It is likely that this rate reflects the low 
immunization rates for RV in Detroit's paediatric population. During June 2006-June 2009, the CDC assessed trends of coverage with $\geqslant 1$ dose of $\mathrm{RV}$ vaccine among infants aged 5 months, and compared RV coverage in the second quarter of 2009 with that of DTaP and PCV-7 at eight participating sentinel sites. RV mean coverage was $72 \%$ but was $13 \%$ lower than mean coverage with $\geqslant 1$ dose of DTaP and PCV-7. In Michigan, the mean sitespecific RV coverage was $76 \%$, compared with $92 \%$ for $\mathrm{DTaP}$ and $91 \%$ for PCV-7. However, Detroit was not one of Michigan's sentinel areas (CDC, 2010) and is traditionally lower than most outlying areas in measures of new vaccine coverage.

Although low RV immunization rates may reflect typical new vaccine coverage dynamics (CDC, 2010), the very low rates seen in our patients indicate that significant barriers to routine RV immunization exist. Identification of these barriers and educating families and providers about the benefits of RV immunization will help in reducing severe $\mathrm{RV}$ disease.

The severity of RV gastroenteritis did not differ significantly in children infected with different genotypes in our study. Children with mixed infection with G1 and G9 had higher severity scores than those infected with any other single genotype, although this difference was not significant. Cascio et al. (2001) have shown that RV strains with a short electropherotype, G2P[4] and subgroup 1 were associated with more severe gastroenteritis symptoms than strains associated with long electropherotypes, G1P[8], G4P[8] and subgroup 2. The authors hypothesized that this association might be due to reintroduction of a G2 serotype, which had not circulated before, in a population that was immunologically naïve to this serotype (Cascio et al., 2001). In a study of hospitalized children in France that was conducted in parallel with emergence of G9 strains, no difference in severity between G1P[8] and G9P[8] infections was found (Aupiais et al., 2009). Similarly, no difference in clinical presentation or severity was found among different $\mathrm{G}$ genotypes in another more recent study (de Rougemont et al., 2011). However, correlation between severity and the infecting genotype in our study should be interpreted with caution because of the small and variable number of patients in different groups and the possible bias in only selecting patients hospitalized due to severe disease.

Our results support the need for continuous surveillance of $\mathrm{RV}$ strains to monitor the prevalence of $\mathrm{G}$ and $\mathrm{P}$ types. The increased prevalence of G9 serotypes in our community and its association with different $\mathrm{P}$ types, including $\mathrm{P}[6]$, raises the possibility that G9 may represent a re-emerging strain that could escape vaccine-induced immunity and become more prevalent following wide use of the new RV vaccines.

In conclusion, there is a diversity of RV strains circulating in the metropolitan Detroit area, including a high proportion of genotype G9 which is not part of the current vaccine formulations. The majority of our study patients were not RV-vaccinated. However, the protective efficacy of RV vaccines and their effect on the prevalence of G9 strains or on the emergence of other RV strains remain to be determined.

\section{ACKNOWLEDGEMENTS}

The study was supported by a grant from Children's Research Center of Michigan, Wayne State University. We thank Dr Marilynn Fairfax for editorial assistance and Dr Ronald Thomas for assistance with statistical analysis. This work was presented in part at the Infectious Diseases Society of America Annual Meeting, Philadelphia, PA, USA, 29 October-1 November 2009.

\section{REFERENCES}

Abdel-Haq, N. M., Thomas, R. A., Asmar, B. I., Zacharova, V. \& Lyman, W. D. (2003). Increased prevalence of G1P[4] genotype among children with rotavirus-associated gastroenteritis in metropolitan Detroit. J Clin Microbiol 41, 2680-2682.

Ansaldi, F., Pastorino, B., Valle, L., Durando, P., Sticchi, L., Tucci, P., Biasci, P., Lai, P., Gasparini, R. \& other authors (2007). Molecular characterization of a new variant of rotavirus $P[8] \mathrm{G} 9$ predominant in a sentinel-based survey in central Italy. J Clin Microbiol 45, 10111015.

Araújo, I. T., Ferreira, M. S., Fialho, A. M., Assis, R. M., Cruz, C. M., Rocha, M. \& Leite, J. P. (2001). Rotavirus genotypes P[4]G9, P[6]G9, and $\mathrm{P}[8] \mathrm{G} 9$ in hospitalized children with acute gastroenteritis in Rio de Janeiro, Brazil. J Clin Microbiol 39, 1999-2001.

Aupiais, C., de Rougemont, A., Menager, C., Vallet, C., Brasme, J. F., Kaplon, J., Pothier, P. \& Gendrel, D. (2009). Severity of acute gastroenteritis in infants infected by G1 or G9 rotaviruses. J Clin Virol 46, 282-285.

Cascio, A., Vizzi, E., Alaimo, C. \& Arista, S. (2001). Rotavirus gastroenteritis in Italian children: can severity of symptoms be related to the infecting virus? Clin Infect Dis 32, 1126-1132.

CDC (2008). Rotavirus surveillance - worldwide, 2001-2008. MMWR Morb Mortal Wkly Rep 57, 1255-1257.

CDC (2010). Rotavirus vaccination coverage among infants aged 5 months - immunization information system sentinel sites, United States, June 2006-June 2009. MMWR Morb Mortal Wkly Rep 59, 521524.

Clark, H. F., Hoshino, Y., Bell, L. M., Groff, J., Hess, G., Bachman, P. \& Offit, P. A. (1987). Rotavirus isolate WI61 representing a presumptive new human serotype. J Clin Microbiol 25, 1757-1762.

Cunliffe, N. A., Gondwe, J. S., Graham, S. M., Thindwa, B. D., Dove, W., Broadhead, R. L., Molyneux, M. E. \& Hart, C. A. (2001). Rotavirus strain diversity in Blantyre, Malawi, from 1997 to 1999. J Clin Microbiol 39, 836-843.

de Rougemont, A., Kaplon, J., Lebon, P., Huet, F., Denis, F., Alain, S., Fourcade, L., Grosjean, J., El-Hajje, M. J. \& other authors (2009). Unexpected substitution of dominant rotavirus $G$ genotypes in French hospitalized children over five consecutive seasons. Eur J Clin Microbiol Infect Dis 28, 403-407.

de Rougemont, A., Kaplon, J., Pillet, S., Mory, O., Gagneur, A., Minoui-Tran, A., Meritet, J. F., Mollat, C., Lorrot, M. \& other authors (2011). Molecular and clinical characterization of rotavirus from diarrheal infants admitted to pediatric emergency units in France. Pediatr Infect Dis J 30, 118-124. 
Endara, P., Trueba, G., Solberg, O. D., Bates, S. J., Ponce, K., Cevallos, W., Matthijnssens, J. \& Eisenberg, J. N. (2007). Symptomatic and subclinical infection with rotavirus P[8]G9, rural Ecuador. Emerg Infect Dis 13, 574-580.

Esteban, L. E., Rota, R. P., Gentsch, J. R., Jiang, B., Esona, M., Glass, R. I., Glikmann, G. \& Castello, A. A. (2010). Molecular epidemiology of group A rotavirus in Buenos Aires, Argentina 2004-2007: reemergence of G2P [4] and emergence of G9P[8] strains. J Med Virol 82, 1083-1093.

Estes, M. K. \& Kapikian, A. Z. (2007). Rotaviruses. In Fields Virology, pp. 1917-1974. Edited by D. M. Knipe \& P. M. Howley. Philadelphia: Lippincott Williams \& Wilkins.

Gentsch, J. R., Glass, R. I., Woods, P., Gouvea, V., Gorziglia, M., Flores, J., Das, B. K. \& Bhan, M. K. (1992). Identification of group A rotavirus gene 4 types by polymerase chain reaction. J Clin Microbiol 30, 1365-1373.

Gentsch, J. R., Laird, A. R., Bielfelt, B., Griffin, D. D., Banyai, K., Ramachandran, M., Jain, V., Cunliffe, N. A., Nakagomi, O. \& other authors (2005). Serotype diversity and reassortment between human and animal rotavirus strains: implications for rotavirus vaccine programs. J Infect Dis 192 (Suppl. 1), S146-S159.

Gentsch, J. R., Hull, J. J., Teel, E. N., Kerin, T. K., Freeman, M. M., Esona, M. D., Griffin, D. D., Bielfelt-Krall, B. P., Banyai, K. \& other authors (2009). G and $P$ types of circulating rotavirus strains in the United States during 1996-2005: nine years of prevaccine data. J Infect Dis 200 (Suppl. 1), S99-S105.

Gouvea, V., Glass, R. I., Woods, P., Taniguchi, K., Clark, H. F., Forrester, B. \& Fang, Z. Y. (1990). Polymerase chain reaction amplification and typing of rotavirus nucleic acid from stool specimens. J Clin Microbiol 28, 276-282.

Gurgel, R. Q., Cuevas, L. E., Vieira, S. C., Barros, V. C., Fontes, P. B., Salustino, E. F., Nakagomi, O., Nakagomi, T., Dove, W. \& other authors (2007). Predominance of rotavirus P[4]G2 in a vaccinated population, Brazil. Emerg Infect Dis 13, 1571-1573.

Iturriza-Gómara, M., Green, J., Brown, D. W., Ramsay, M., Desselberger, U. \& Gray, J. J. (2000). Molecular epidemiology of human group A rotavirus infections in the United Kingdom between 1995 and 1998. J Clin Microbiol 38, 4394-4401.

Iturriza-Gómara, M., Kang, G. \& Gray, J. (2004). Rotavirus genotyping: keeping up with an evolving population of human rotaviruses. J Clin Virol 31, 259-265.

Koshimura, Y., Nakagomi, T. \& Nakagomi, O. (2000). The relative frequencies of $\mathrm{G}$ serotypes of rotaviruses recovered from hospitalized children with diarrhea: a 10-year survey (1987-1996) in Japan with a review of globally collected data. Microbiol Immunol 44, 499510.

Laird, A. R., Gentsch, J. R., Nakagomi, T., Nakagomi, O. \& Glass, R. I. (2003). Characterization of serotype G9 rotavirus strains isolated in the United States and India from 1993 to 2001. J Clin Microbiol 41, 3100-3111.

Leite, J. P., Alfieri, A. A., Woods, P. A., Glass, R. I. \& Gentsch, J. R. (1996). Rotavirus $G$ and $P$ types circulating in Brazil: characterization by RT-PCR, probe hybridization, and sequence analysis. Arch Virol 141, 2365-2374.

Martella, V., Terio, V., Arista, S., Elia, G., Corrente, M., Madio, A., Pratelli, A., Tempesta, M., Cirani, A. \& Buonavoglia, C. (2004). Nucleotide variation in the VP7 gene affects PCR genotyping of G9 rotaviruses identified in Italy. J Med Virol 72, 143-148.
Parashar, U. D., Hummelman, E. G., Bresee, J. S., Miller, M. A. \& Glass, R. I. (2003). Global illness and deaths caused by rotavirus disease in children. Emerg Infect Dis 9, 565-572.

Parashar, U. D., Gibson, C. J., Bresse, J. S. \& Glass, R. I. (2006). Rotavirus and severe childhood diarrhea. Emerg Infect Dis 12, 304-306. Perez-Schael, I., Blanco, M., Vilar, M., Garcia, D., White, L., Gonzalez, R., Kapikian, A. Z. \& Flores, J. (1990). Clinical studies of a quadrivalent rotavirus vaccine in Venezuelan infants. J Clin Microbiol 28, 553-558.

Ramachandran, M., Gentsch, J. R., Parashar, U. D., Jin, S., Woods, P. A., Holmes, J. L., Kirkwood, C. D., Bishop, R. F., Greenberg, H. B. \& other authors (1998). Detection and characterization of novel rotavirus strains in the United States. J Clin Microbiol 36, 3223-3229.

Ramachandran, M., Kirkwood, C. D., Unicomb, L., Cunliffe, N. A., Ward, R. L., Bhan, M. K., Clark, H. F., Glass, R. I. \& Gentsch, J. R. (2000). Molecular characterization of serotype G9 rotavirus strains from a global collection. Virology 278, 436-444.

Rodrigues, F., Iturriza, M., Gray, J., Januário, L. \& Lemos, L. (2007). Epidemiology of rotavirus in Portugal: G9 as a major cause of diarrhoea in non-hospitalised children. J Clin Virol 40, 214-217.

Ruiz-Palacios, G. M., Pérez-Schael, I., Velázquez, F. R., Abate, H., Breuer, T., Clemens, S. C., Cheuvart, B., Espinoza, F., Gillard, P. \& other authors (2006). Safety and efficacy of an attenuated vaccine against severe rotavirus gastroenteritis. N Engl J Med 354, 11-22.

Samajdar, S., Ghosh, S., Chawla-Sarkar, M., Mitra, U., Dutta, P., Kobayashi, N. \& Naik, T. N. (2008). Increase in prevalence of human group A rotavirus G9 strains as an important VP7 genotype among children in eastern India. J Clin Virol 43, 334-339.

Sánchez-Fauquier, A., Montero, V., Moreno, S., Solé, M., Colomina, J., Iturriza-Gomara, M., Revilla, A., Wilhelmi, I., Gray, J. \& Gegavi/ VIGESS-Net Group (2006). Human rotavirus G9 and G3 as major cause of diarrhea in hospitalized children, Spain. Emerg Infect Dis 12, 1536-1541.

Santos, N. \& Hoshino, Y. (2005). Global distribution of rotavirus serotypes/genotypes and its implication for the development and implementation of an effective rotavirus vaccine. Rev Med Virol 15, 29-56.

Santos, N., Volotão, E. M., Soares, C. C., Albuquerque, M. C., da Silva, F. M., de Carvalho, T. R., Pereira, C. F., Chizhikov, V. \& Hoshino, Y. (2001). Rotavirus strains bearing genotype G9 or P[9] recovered from Brazilian children with diarrhea from 1997 to 1999. J Clin Microbiol 39, 1157-1160.

Santos, N., Volotão, E. M., Soares, C. C., Campos, G. S., Sardi, S. I. \& Hoshino, Y. (2005). Predominance of rotavirus genotype G9 during the 1999, 2000, and 2002 seasons among hospitalized children in the city of Salvador, Bahia, Brazil: implications for future vaccine strategies. J Clin Microbiol 43, 4064-4069.

Steele, A. D. \& Ivanoff, B. (2003). Rotavirus strains circulating in Africa during 1996-1999: emergence of G9 strains and P[6] strains. Vaccine 21, 361-367.

Velázquez, F. R., Matson, D. O., Calva, J. J., Guerrero, L., Morrow, A. L., Carter-Campbell, S., Glass, R. I., Estes, M. K., Pickering, L. K. \& RuizPalacios, G. M. (1996). Rotavirus infections in infants as protection against subsequent infections. N Engl J Med 335, 1022-1028.

Vesikari, T., Matson, D. O., Dennehy, P., Van Damme, P., Santosham, M., Rodriguez, Z., Dallas, M. J., Heyse, J. F., Goveia, M. G. \& other authors (2006). Safety and efficacy of a pentavalent human-bovine (WC3) reassortant rotavirus vaccine. $N$ Engl J Med 354, 23-33. 